\title{
Collective structural evolution in neutron-rich Yb, Hf, W, Os, and Pt isotopes
}

\author{
K. Nomura, ${ }^{1}$ T. Otsuka, ${ }^{1,2,3}$ R. Rodríguez-Guzmán, ${ }^{4}$ L. M. Robledo, ${ }^{5}$ and P. Sarriguren ${ }^{4}$ \\ ${ }^{1}$ Department of Physics, University of Tokyo, Hongo, Bunkyo-ku, Tokyo 113-0033, Japan \\ ${ }^{2}$ Center for Nuclear Study, University of Tokyo, Hongo, Bunkyo-ku, Tokyo 113-0033, Japan \\ ${ }^{3}$ National Superconducting Cyclotron Laboratory, Michigan State University, East Lansing, Michigan 48824-1321, USA \\ ${ }^{4}$ Instituto de Estructura de la Materia, IEM-CSIC, Serrano 123, E-28006 Madrid, Spain \\ ${ }^{5}$ Departamento de Física Teórica, Universidad Autónoma de Madrid, E-28049 Madrid, Spain \\ (Received 19 April 2011; revised manuscript received 19 October 2011; published 21 November 2011)
}

\begin{abstract}
An interacting-boson-model Hamiltonian determined from Hartree-Fock-Bogoliubov calculations with the microscopic Gogny energy density functional D1M is applied to the spectroscopic analysis of neutron-rich $\mathrm{Yb}, \mathrm{Hf}$, W, Os, and Pt isotopes with mass $A \sim 180-200$. Excitation energies and transition rates for the relevant low-lying quadrupole collective states are calculated by this method. Transitions from prolate to oblate ground-state shapes are analyzed as a function of neutron number $N$ in a given isotopic chain by calculating excitation energies, $B(E 2)$ ratios, and correlation energies in the ground state. It is shown that such transitions tend to occur more rapidly for the isotopes with lower proton number $Z$ when departing from the proton shell closure $Z=82$. The triaxial degrees of freedom turn out to play an important role in describing the considered mass region. Predicted low-lying spectra for the neutron-rich exotic $\mathrm{Hf}$ and $\mathrm{Yb}$ isotopes are presented. The approximations used in the model and the possibilities to refine its predictive power are addressed.
\end{abstract}

DOI: 10.1103/PhysRevC.84.054316

PACS number(s): 21.10.Re, 21.60.Ev, 21.60.Fw, 21.60.Jz

\section{INTRODUCTION}

The study of the origin of nuclear deformation and its evolution as a function of proton and neutron numbers has attracted considerable theoretical interest from a large variety of viewpoints [1-13]. Experimentally, low-lying spectroscopy provides a very powerful source of information that allows one to establish signatures correlating the nuclear shape evolution with the energy spectra [14-23].

Among many other nuclear structure models, selfconsistent mean-field methods, based on microscopic energy density functionals (EDFs), have provided both accurate and universal descriptions of different nuclear intrinsic properties including binding energies, ground-state deformations, density distributions, low-lying one-quasi-particle configurations, as well as the way nuclear shapes evolve with the number of nucleons [2,6,8-11,24-28]. Popular EDFs are the nonrelativistic Skyrme [6,29,30] and Gogny [31,32] ones, as well as relativistic mean-field Lagrangians [33]. To describe nuclear spectroscopy one should go beyond the mean-field approximation to take into account the restorations of broken symmetries and/or the configuration mixing of intrinsic states in the spirit of the generator coordinate method (GCM) [2,6,34-38]. In these kinds of studies, calculations may become computationally much more demanding and time consuming than the underlying mean field, particularly when triaxial degrees of freedom are included in the analysis.

A sound approximation to the full GCM configuration mixing and/or the symmetry restoration is the five-dimensional collective Hamiltonian with quadrupole degrees of freedom where both rotational and vibrational mass parameters are determined from the constrained, self-consistent mean-field calculations with a given EDF and the collective potential is derived by the zero-point energy correction to the total mean-field energy (e.g., Refs. [39-41]).
Alternatively, nuclear dynamics and spectroscopic quantities can be approximated by introducing appropriate bosonic degrees of freedom. The interacting boson model (IBM) [42] can be regarded as a nice example for this, and it has been exploited in a large number of phenomenological studies focusing on the low-lying spectrum of medium-heavy and heavy nuclei [42]. The simplest version of the IBM is built on monopole $s$ and quadrupole $d$ bosons, which reflect the collective $J^{\pi}=0^{+}$and $2^{+}$pairs of valence-shell configurations, respectively [43]. Nevertheless, because the IBM itself should have a certain microscopic foundation, a Hamiltonian of the IBM was derived conventionally from the shell-model configuration [43] and more recently from EDF-based calculations [44]. These mapping methods have been applied to realistic cases involving a variety of situations, from nuclei with modest quadrupole deformation including $\gamma$-unstable ones [43-47], to strongly deformed rotational nuclei [48-53]. Also quantum-mechanical correlation effects in the ground state have been considered [46]. Starting from the constrained Hartree-Fock-Bogoliubov (HFB) theory with the D1S [54] parametrization of the Gogny functional, the method of Ref. [44] was used for the spectroscopic analysis of $\mathrm{Pt}$ isotopes [47], and some Os and W isotopes [53].

In this paper we apply the mapping procedure of Ref. [44] to the mass region $A \sim 180-200$, extending the analysis made in Refs. [47,53] to the neighboring exotic $\mathrm{Hf}$ and $\mathrm{Yb}$ nuclei. An additional motivation is to explore some possibilities to refine the predictive power of the method for the considered mass region. Although the D1S parametrization of the Gogny force is considered a global EDF able to describe many low-energy nuclear data with reasonable predictive power (see, for example, Refs. [41,55,56] and references therein), in this paper we preferred to use the Gogny-D1M functional [57]. Systematic explorations of different nuclear phenomena $[55,57]$, including properties of odd nuclei computed within 
the equal-filling approximation [24-26], suggest that the new parametrization of the Gogny-EDF is as good as the standard D1S, a fact that we intend to confirm in this paper.

It is well known that the nuclei in the mass region $A \sim$ 180-200 exhibit a transition between prolate and oblate equilibrium shapes as a function of the nucleon number, with the critical point around $N \approx 116$ having a pronounced $\gamma$ softness [58-61]. These facts make the region a potential testing ground to understand the deformation properties of atomic nuclei. The evolution of the nuclear ground states in this mass region was investigated recently with the constrained self-consistent mean-field method with microscopic EDFs $[9,56,62]$. Both the (constrained) Hartree-Fock + BCS (HF + BCS) and the HFB approximations were used to compute energy surfaces with quadrupole degrees of freedom in order to give a microscopic insight into shape transitions $[9,55,56]$. It was shown in these studies that the triaxiality is an important ingredient to describe the evolution from prolate to oblate shapes, irrespective of the types of EDFs used.

It should be kept in mind that $\mathrm{Pt}, \mathrm{Hg}$, and $\mathrm{Pb}$ isotopes are well known [63] for the spectacular coexistence of different low-lying configurations based on different intrinsic deformations as observed in their low-lying spectrum. There are a number of works aimed at understanding the shape coexistence phenomenon in this region in terms of both EDF-based microscopic calculations $[36,64,65]$ and phenomenological models [66-68].

The paper is organized as follows: In Sec. II, a short outline of the theoretical framework is given. Section III presents the energy surfaces, ground-state correlation energies, moments of inertia for the rotational bands, low-lying spectra, and the $B(E 2)$ systematics for the considered isotopes chains. Section IV is devoted to the concluding remarks and work perspectives.

\section{THEORETICAL PROCEDURE}

The analysis starts with a constrained HFB calculation using the Gogny-D1M EDF. The constraints in this case refer to the mass quadrupole moments which are associated with the quadrupole deformation parameters $\beta$ and $\gamma$ in the geometrical model [1]. The set of constrained HFB calculations, for each collective coordinate $(\beta, \gamma)$, provides the total HFB energy [denoted by $E_{\mathrm{HFB}}(\beta, \gamma)$ ]. For calculation details the reader is referred to Refs. [47,56].

In other studies solving the five-dimensional collective Hamiltonian [39-41], the collective potential energy surface is obtained by subtracting the zero-point energies for both rotational and vibrational motions from the constrained HFB energy surface. This corrected energy surface should be viewed as a collective potential energy surface. In the present work, the constrained HFB energy surface and the corresponding boson energy surface are compared, and they are referred to simply as the energy surface. Note that, because the total energy is considered, all ingredients, including those relevant to kinetic terms, are supposed to be taken into account to a good extent.

Each point of the Gogny-HFB energy surface $E_{\mathrm{HFB}}(\beta, \gamma)$ is mapped onto the corresponding point on the bosonic energy surface, denoted by $E_{\mathrm{IBM}}\left(\beta_{B}, \gamma_{B}\right)$, with $\beta_{B}$ and $\gamma_{B}$ being the deformation parameters for the boson system, in such a way that the bosonic energy surface fits the fermionic one [44]. In this paper we consider the proton-neutron interacting boson model (IBM-2) [43] because it reflects better the microscopic picture than the original version of the IBM without distinction of the proton and the neutron degrees of freedom (often called IBM-1). In what follows we denote the IBM-2 simply as the IBM, unless otherwise specified. The IBM energy surface is obtained as the expectation value of a given boson Hamiltonian [69] in terms of the coherent state $\left|\Phi\left(\beta_{B}, \gamma_{B}\right)\right\rangle$. The coherent state represents the intrinsic wave function of the boson system and is characterized by the deformation variables $\beta_{B}$ and $\gamma_{B}$. In principle, proton and neutron bosons might have different values of the deformation parameters, but because proton and neutron systems are supposed to attract each other strongly in medium-heavy and heavy deformed nuclei, the deformations of proton and neutron systems can be taken the same to a good approximation.

If the separability of the mapping along the $\beta$ and $\gamma$ directions is assumed, one can consider the relation between the IBM and the geometrical deformation variables [44,46]. It was shown [69] that, in general terms, the bosonic and the geometrical $\beta$ 's are proportional to each other and that the proportionality coefficient coincides with the ratio of the total nucleon number to the valence nucleon number counted from the nearest closed shells. We exploit this relation and assume that $\beta_{\mathrm{B}}=C_{\beta} \beta$, with $C_{\beta}$ being a numerical coefficient [44]. The typical range of the $C_{\beta}$ value turns out to be $\sim 5-10$, which is about the same order of magnitude as the actual ratios of the total nucleon number to the valence nucleon number. Regarding the triaxial parameter $\gamma$, the identification $\gamma_{\mathrm{B}}=\gamma$ seems valid as indeed both geometrical and IBM $\gamma$ 's have the same meaning, ranging from $0^{\circ}$ to $60^{\circ}$.

We adopt the IBM Hamiltonian of the following form:

$$
\hat{H}_{\mathrm{IBM}}=\epsilon \hat{n}_{d}+\kappa \hat{Q}_{\pi} \cdot \hat{Q}_{\nu}+\alpha \hat{L} \cdot \hat{L},
$$

where the first term $\hat{n}_{d}=\hat{n}_{d \pi}+\hat{n}_{d \nu}$ with $\hat{n}_{d \rho}=d_{\rho}^{\dagger} \cdot \tilde{d}_{\rho}(\rho=$ $\pi$ or $v$ ) is identified as the $d$-boson number operator. The second term on the right-hand side of Eq. (1) stands for the quadrupole-quadrupole interaction between proton and neutron systems, with $\hat{Q}_{\rho}=s_{\rho}^{\dagger} \tilde{d}_{\rho}+d_{\rho}^{\dagger} \tilde{s}_{\rho}+\chi_{\rho}\left[d_{\rho}^{\dagger} \tilde{d}_{\rho}\right]^{(2)}$ being the quadrupole operator for proton or neutron systems. The third term (denoted the LL term hereafter) is relevant to the moment of inertia of the rotational band. $\hat{L}=\hat{L}_{\pi}+\hat{L}_{v}$ is the angular momentum operator for the boson system with $\hat{L}_{\rho}=\sqrt{10}\left[d_{\rho}^{\dagger} \tilde{d}_{\rho}\right]^{(1)}$.

The form of the Hamiltonian $\hat{H}_{\mathrm{IBM}}$ in Eq. (1) is not the most general, but it embodies all essential features of the low-lying quadrupole collective states. A more general IBM Hamiltonian with up to two-body interactions contains many more terms than those considered here. However, these additional terms are supposed to be of little importance, and their implementation would increase the number of parameters, which makes the problem quite complicated.

The parameters contained in the first two terms of the Hamiltonian $\hat{H}_{\text {IBM }}$ in Eq. (1), $\epsilon, \kappa, \chi_{\pi}$, and $\chi_{\nu}$, as well as the coefficient $C_{\beta}$, are fixed using the fitting method of Ref. [46]. 
The LL term contributes to the energy surface in the same way as the $d$-boson number operator, but with a different coefficient, $6 \alpha$. Hence, the $\alpha$ coefficient cannot be fixed only by the mapping of the energy surface. A further step is required in order to incorporate specific nonzero angular frequency features of the rotational cranking. The $\alpha$ value is determined by the procedure of Ref. [52], where the cranking moment of inertia was compared between fermion and boson systems.

We then calculate the moment of inertia for the $2_{1}^{+}$excited state by the Thouless-Valatin (TV) formula [70],

$$
\mathcal{J}_{\mathrm{TV}}=3 / E_{\gamma} .
$$

Here, $E_{\gamma}$ stands for the $2_{1}^{+}$excitation energy obtained from the self-consistent cranking method with the constraint $\left\langle\hat{J}_{x}\right\rangle=$ $\sqrt{L(L+1)}$, where $\hat{J}_{x}$ represents the $x$ component of the (fermion) angular momentum operator [56]. In Ref. [52], the Inglis-Belyaev formula [71,72] turned out to be valid for the rotational regime, but the present $\mathrm{TV}$ moment of inertia appears to be more general.

For the boson system, we calculate the moment of inertia of the intrinsic (coherent) state, denoted by $\mathcal{J}_{\text {IBM }}$, using the cranking formula of Ref. [73],

$$
\mathcal{J}_{\mathrm{IBM}}\left(\beta_{B}, \gamma_{B}\right)=\lim _{\omega \rightarrow 0} \frac{1}{\omega} \frac{\left\langle\Phi\left(\beta_{B}, \gamma_{B}\right)\left|\hat{L}_{x}\right| \Phi\left(\beta_{B}, \gamma_{B}\right)\right\rangle}{\left\langle\Phi\left(\beta_{B}, \gamma_{B}\right) \mid \Phi\left(\beta_{B}, \gamma_{B}\right)\right\rangle},
$$

where $\omega$ and $\hat{L}_{x}$ stand for the cranking frequency and the $x$ component of the boson angular momentum operator, respectively.

While $\mathcal{J}_{\text {IBM }}$ has six parameters $\epsilon, \kappa, \chi_{\pi}, \chi_{\nu}, C_{\beta}$, and $\alpha$, all of them but $\alpha$ are already fixed by the energy-surface analysis. The $\alpha$ value for each nucleus is obtained so that the $\mathcal{J}_{\text {IBM }}$ value at the equilibrium point, where the boson energy surface $E_{\mathrm{IBM}}\left(\beta_{B}, \gamma_{B}\right)$ is minimal, becomes identical to the $\mathcal{J}_{\mathrm{TV}}$ value at its corresponding energy minimum.

The values of all derived IBM parameters are summarized in Table I. When diagonalizing the Hamiltonian in Eq. (1), the $\epsilon$ parameter is shifted by $\Delta \epsilon=6 \alpha$. The $\epsilon$ value listed in Table I is the one with this shift.

The diagonalization of the IBM Hamiltonian, which is parametrized by the set of interaction strengths summarized in Table I, generates the energies and the wave functions of the excited states. Diagonalization is performed in the boson $M$-scheme basis, where $M$ denotes the $z$ component of the boson angular momentum operator. With the eigenvectors of the Hamiltonian $\hat{H}_{\mathrm{IBM}}$, the $B(E 2)$ value is calculated:

$$
B\left(E 2 ; L \rightarrow L^{\prime}\right)=\frac{1}{2 L+1} \mid\left\langle\left. L^{\prime}\left\|\hat{T}^{(E 2)}\right\| L\right|^{2},\right.
$$

where $L$ and $L^{\prime}$ are the angular momenta for the initial and the final states, respectively. In the present work the $E 2$ operator is given as $\hat{T}^{(E 2)}=e_{\pi} \hat{Q}_{\pi}+e_{\nu} \hat{Q}_{\nu}$, where $\hat{Q}_{\rho}$ coincides with the quadrupole operator in Eq. (1), and thus the same values of the $\chi_{\pi}$ and $\chi_{v}$ parameters as those listed in Table I are used in calculating the $B(E 2)$ values [so-called consistent- $Q$ formalism (cf. Ref. [42])]. The boson effective charges for protons and neutrons are taken the same, namely $e_{\pi}=e_{\nu}$.
TABLE I. The parameters for the IBM Hamiltonian $\hat{H}_{\text {IBM }}$ of Eq. (1), as well as the coefficient $C_{\beta}$, obtained from the mapping

\begin{tabular}{|c|c|c|c|c|c|c|}
\hline & $\begin{array}{c}\epsilon \\
(\mathrm{keV})\end{array}$ & $\begin{array}{c}-\kappa \\
(\mathrm{keV})\end{array}$ & $\begin{array}{c}\chi_{\pi} \\
\left(\times 10^{3}\right)\end{array}$ & $\begin{array}{c}\chi_{v} \\
\left(\times 10^{3}\right)\end{array}$ & $\begin{array}{c}\alpha \\
(\mathrm{keV})\end{array}$ & $C_{\beta}$ \\
\hline${ }^{180} \mathrm{Yb}$ & 212 & 265 & 337 & -991 & -9.06 & 3.60 \\
\hline${ }^{182} \mathrm{Yb}$ & 169 & 265 & 300 & -900 & -11.4 & 3.70 \\
\hline${ }^{184} \mathrm{Yb}$ & 279 & 271 & 302 & -548 & -9.84 & 3.87 \\
\hline${ }^{186} \mathrm{Yb}$ & 418 & 268 & 147 & -106 & -9.54 & 4.90 \\
\hline${ }^{188} \mathrm{Yb}$ & 528 & 265 & 418 & 43 & -4.68 & 5.13 \\
\hline${ }^{190} \mathrm{Yb}$ & 769 & 267 & 332 & 573 & -0.185 & 5.50 \\
\hline${ }^{192} \mathrm{Yb}$ & 806 & 271 & 461 & 862 & 21.5 & 7.20 \\
\hline${ }^{182} \mathrm{Hf}$ & 124 & 280 & 489 & -913 & -5.61 & 3.93 \\
\hline${ }^{184} \mathrm{Hf}$ & 128 & 282 & 458 & -938 & -8.01 & 4.07 \\
\hline${ }^{186} \mathrm{Hf}$ & 109 & 275 & 400 & -700 & -4.85 & 4.40 \\
\hline${ }^{188} \mathrm{Hf}$ & 250 & 277 & 282 & -208 & -7.90 & 5.30 \\
\hline${ }^{190} \mathrm{Hf}$ & 442 & 280 & 403 & -30 & -5.99 & 5.48 \\
\hline${ }^{192} \mathrm{Hf}$ & 619 & 273 & 388 & 443 & 2.79 & 5.94 \\
\hline${ }^{194} \mathrm{Hf}$ & 716 & 277 & 534 & 805 & 18.4 & 8.20 \\
\hline${ }^{184} \mathrm{~W}$ & 50.4 & 286 & 409 & -859 & -0.400 & 4.09 \\
\hline${ }^{186} \mathrm{~W}$ & 36.8 & 285 & 389 & -835 & -2.30 & 4.50 \\
\hline${ }^{188} \mathrm{~W}$ & 69.6 & 289 & 401 & -662 & -1.44 & 4.80 \\
\hline${ }^{190} \mathrm{~W}$ & 71.3 & 275 & 572 & -419 & -2.72 & 5.60 \\
\hline${ }^{192} \mathrm{~W}$ & 231 & 270 & 189 & 147 & -4.15 & 6.30 \\
\hline${ }^{194} \mathrm{~W}$ & 627 & 291 & 392 & 536 & -5.74 & 6.87 \\
\hline${ }^{196} \mathrm{~W}$ & 686 & 281 & 745 & 822 & 15.3 & 8.50 \\
\hline${ }^{186} \mathrm{Os}$ & 142 & 310 & 331 & -689 & -0.433 & 4.40 \\
\hline${ }^{188} \mathrm{Os}$ & 162 & 318 & 352 & -672 & -2.78 & 4.83 \\
\hline${ }^{190} \mathrm{Os}$ & 86.7 & 303 & 412 & -509 & -2.61 & 5.40 \\
\hline${ }^{192} \mathrm{Os}$ & 91.5 & 292 & 502 & -488 & -3.09 & 6.15 \\
\hline${ }^{194}$ Os & 289 & 305 & 401 & -77 & -6.04 & 6.74 \\
\hline${ }^{196} \mathrm{Os}$ & 541 & 298 & 336 & 513 & -5.94 & 7.64 \\
\hline${ }^{198} \mathrm{Os}$ & 683 & 304 & 573 & 793 & 8.50 & 9.66 \\
\hline${ }^{188} \mathrm{Pt}$ & 187 & 328 & 409 & -487 & 8.16 & 4.81 \\
\hline${ }^{190} \mathrm{Pt}$ & 215 & 336 & 300 & -10 & 5.93 & 5.56 \\
\hline${ }^{192} \mathrm{Pt}$ & 311 & 362 & 265 & 44 & -0.117 & 6.44 \\
\hline${ }^{194} \mathrm{Pt}$ & 312 & 366 & 490 & -50 & 0.214 & 6.85 \\
\hline${ }^{196} \mathrm{Pt}$ & 435 & 356 & 475 & 311 & 1.87 & 7.28 \\
\hline${ }^{198} \mathrm{Pt}$ & 489 & 319 & 611 & 565 & 8.80 & 7.90 \\
\hline${ }^{200} \mathrm{Pt}$ & 719 & 308 & 467 & 949 & -4.69 & 8.78 \\
\hline
\end{tabular}
of HFB to IBM energy surfaces for the considered Yb, Hf, W, Os, and Pt nuclei with $N=110-122$.

\section{RESULTS AND DISCUSSION}

\section{A. Energy surfaces}

Figure 1 shows the mapped IBM energy surfaces for $\mathrm{Yb}$, $\mathrm{Hf}, \mathrm{W}, \mathrm{Os}$, and Pt isotopes with $112 \leqslant N \leqslant 120$. Each energy surface is plotted in terms of $\beta\left(=\beta_{B} / C_{\beta}\right)$ and $\gamma\left(=\gamma_{B}\right)$ up to $2 \mathrm{MeV}$ from its absolute minimum, because most of the quadrupole collective states are within this range. Note that the IBM energy surfaces for $N=110$ and 122 are not drawn because they are similar to those for $N=112$ and 120 nuclei, respectively. The Gogny-D1M energy surfaces are not shown because they do not differ substantially from the ones depicted in Ref. [56] with Gogny-D1S

For all the isotopes but the Pt ones, the energy minimum shifts from the prolate $\left(\gamma=0^{\circ}\right)$ to the oblate $\left(\gamma=60^{\circ}\right)$ sides 


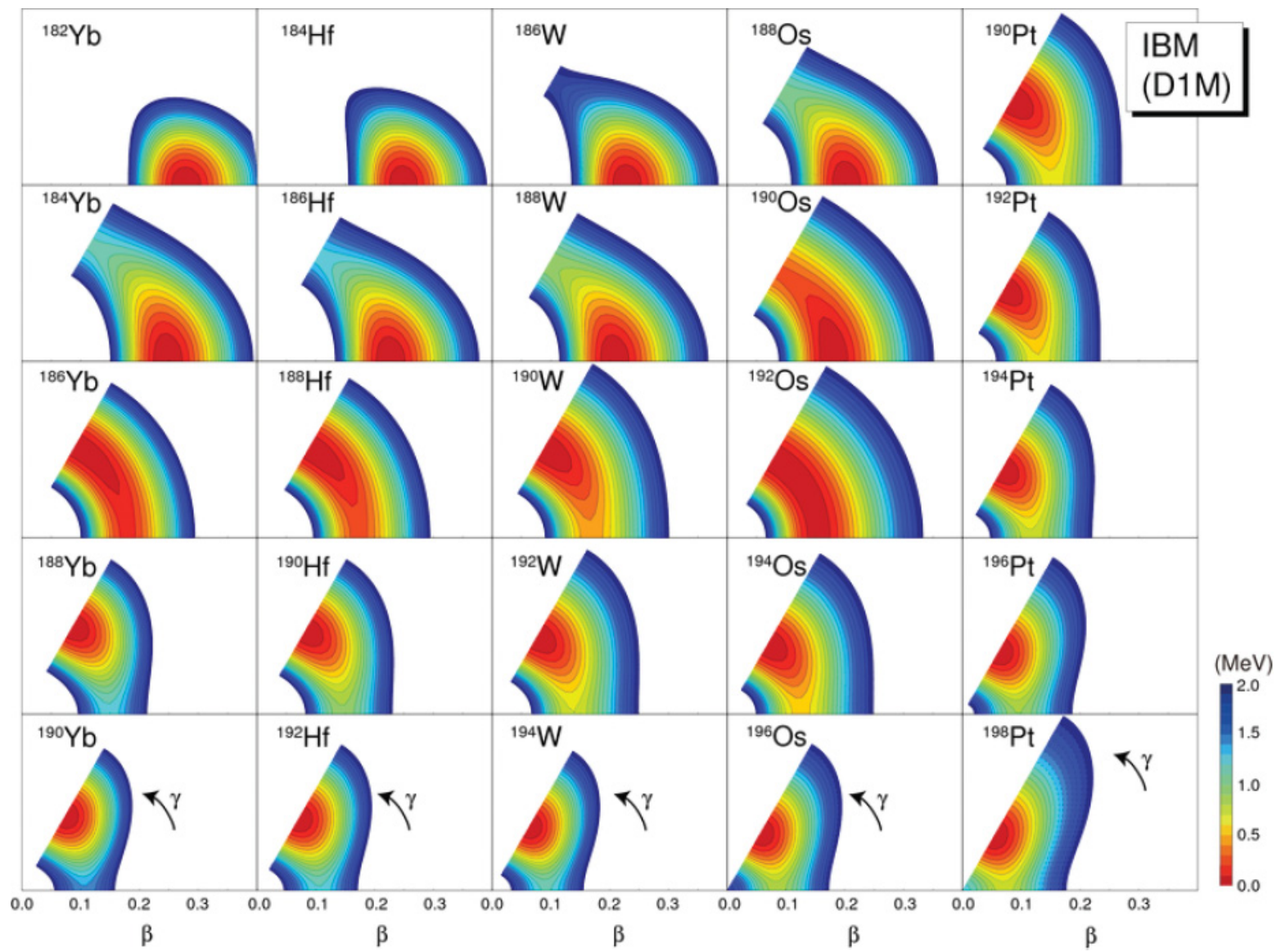

FIG. 1. (Color online) The IBM energy surfaces for the considered Yb, Hf, W, Os, and Pt isotopes with $N=112-120$, obtained by the mapping from the Gogny-D1M energy surface, depicted within $0 \leqslant \beta \leqslant 0.4$ and $0^{\circ} \leqslant \gamma \leqslant 60^{\circ}$ up to $2 \mathrm{MeV}$ excitation from the minimum. Contour spacing is $100 \mathrm{keV}$.

as the number of neutrons increases, passing through the most notable $\gamma$-soft nuclei with $N \approx 116$. The derived $\chi_{\pi}$ and $\chi_{\nu}$ values for many $N=116$ isotones then satisfy $\chi_{\pi}+\chi_{\nu} \approx 0$, as summarized in Table I. This choice of the $\chi$ parameters is at the origin of the almost totally flat topology of the energy surface in the IBM-2, as seen for example in the ${ }^{192}$ Os nucleus in Fig. 1. The change in the topology of the energy surface is an evidence of prolate-to-oblate shape-phase transition, which becomes sharper for smaller $Z$. The Gogny-D1S energy surfaces reported in Refs. [47,56] were somewhat steeper in both $\beta$ and $\gamma$ directions than the present Gogny-D1M ones.

A difference is apparent between the energy surfaces of the Pt isotopes and those of the others. For the Pt isotopes, the variation of the energy surface takes place much more moderately. Such slow structural transition in Pt isotopes was also observed in the case of the D1S functional $[47,55]$. While a certain quantitative difference is observed between the two Gogny functional results, the conclusion does not change.

It should be noted that the Gogny-HFB calculation suggested shallow triaxial wells for the transitional, $N=116$ Os and W nuclei [56]. In contrast, the mapped IBM energy surfaces in Fig. 1 are flat in the $\gamma$ direction, because the only $\gamma$-dependent term of the bosonic energy surface is proportional to $\cos 3 \gamma$. This is the case as long as the boson Hamiltonian contains up to two-body interactions. Only when a three-body (so-called cubic) term is considered is a stable minimum at a $\gamma$ value different from $\gamma=0^{\circ}$ and $60^{\circ}$ obtained [74,75].

\section{B. Correlation energies}

We next discuss a signature for a shape transition from a simple perspective. To do this we consider the following quantity, which is called the correlation energy hereafter, as already introduced in Ref. [46]:

$$
E_{\mathrm{Corr}}=E_{\mathrm{IBM}}\left(0_{1}^{+}\right)-\left\langle\hat{H}_{\mathrm{IBM}}\right\rangle_{\mathrm{min}},
$$

where the first term $E_{\mathrm{IBM}}\left(0_{1}^{+}\right)$is the eigenenergy of the IBM Hamiltonian, Eq. (1), for the $L^{\pi}=0^{+}$ground state, and the second term $\left\langle\hat{H}_{\mathrm{IBM}}\right\rangle_{\min }$ denotes the minimum value of the IBM energy surface, which is obtained by the variation with respect to $\beta$ and $\gamma$.

In the self-consistent mean-field calculation with a given EDF (e.g., Ref. [34]), the quantum-mechanical effect can be extracted by comparing the minimum value of the total energy surface of the mean field with the $L^{\pi}=0^{+}$eigenenergy resulting from the restoration of the broken symmetries and the configuration mixing. For calculations of correlation energies by mapping the EDF theory into shell-model-like interactions, 


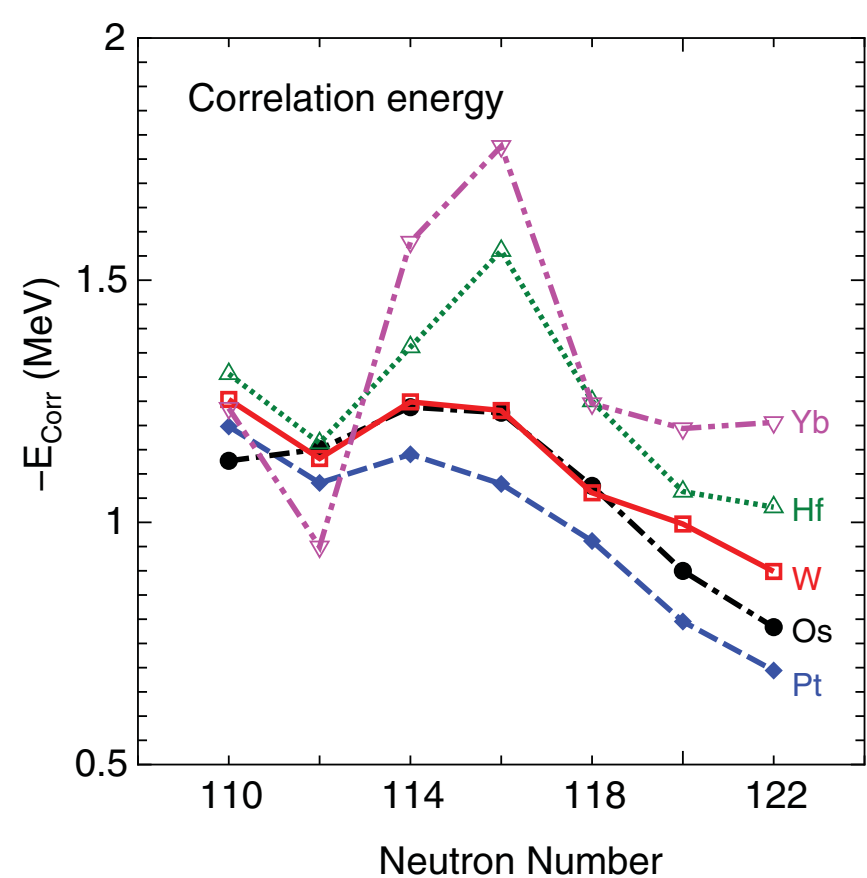

FIG. 2. (Color online) Correlation energy $E_{\text {Corr }}$ defined in Eq. (5) for $\mathrm{Yb}, \mathrm{Hf}, \mathrm{W}$, Os, and $\mathrm{Pt}$ isotopes.

including quadrupole and pairing correlations, the reader is referred to Ref. [76].

In the present study, all correlation effects can be included by the diagonalization of the boson Hamiltonian, and the energies and the wave functions of the states with good angular momentum and particle number can be generated. Thus, the quantity defined in Eq. ( (5)) contains correlation energies coming from symmetry restoration and configuration mixing and is similar to the equivalent quantity discussed in GCM studies.

The behavior of $E_{\text {Corr }}$ with neutron number correlates well with the underlying shape transition. Figure 2 shows that for each considered isotopic chain the correlation energy is maximal in magnitude at the neutron number $N \sim 116$, which corresponds to the transition point of the prolate-to-oblate shape transition, and decreases as the neutron shell closure $N=126$ is approached. This is consistent with the overall systematic trend of the underlying energy surface in Fig. 1. These features were recognized in the GCM studies (e.g., in Ref. [38]) also. For the Pt isotopes, the magnitude of $E_{\text {Corr }}$ decreases with $N$, indicating that a clear transition is not expected for these nuclei.

Compared with the analysis by the GCM configuration mixing using, for example, a Skyrme functional [34] for the same mass region considered here, the magnitude of the present correlation energy $E_{\text {Corr }}$ is rather small, whereas the qualitative features mentioned earlier do not contradict the GCM results.

In comparison to some rare-earth nuclei such as $\mathrm{Nd}-\mathrm{Sm}$ Gd isotopes, where a distinct first-order shape transition is observed [13], the shape transition occurs rather moderately in the considered mass region. Thus, contrary to $E_{\text {Corr }}$ in Fig. 2, any drastic change with nucleon number is not expected in some other quantities in the ground state, such as two-nucleon separation energies.

\section{Moments of inertia}

On the basis of the analysis in Sec. III B, we discuss to what extent the moment of inertia is affected by the configuration mixing due to the diagonalization of the IBM Hamiltonian. The effect is most nicely illustrated in the $\mathrm{W}$ isotopes, for which relatively many experimental spectroscopic data are available.

We show in Fig. 3 the moments of inertia of $\mathrm{W}$ isotopes, calculated by the cranking formula for the coherent state $\mathcal{J}_{\text {IBM }}$ in Eq. (3) and those taken from the $2_{1}^{+}$eigenenergies of the IBM and the experimental $2_{1}^{+}$excitation energies [77] using the rotor formula $L(L+1)$. Note that the cranking moment of inertia of the IBM is, due to the correction by the LL term, set identical to the TV moment of inertia. Thus the TV moment of inertia is not depicted in Fig. 3.

The experimental moment of inertia decreases with $N$ and the slope of this decrease appears to change at $N=116$. This change suggests a gradual shape transition. The moment of inertia of the IBM intrinsic state, in contrast, decreases smoothly with the exception of the kink at $N=114$. Perhaps such a kink reflects a detailed shell structure irrelevant to the present work. However, the kink is eliminated in the moment of inertia after diagonalization, which falls on the same systematics as the experimental data.

It appears that, from Fig. 3, the cranking moment of inertia still works for the nuclei $N=110$ and 112 , for which one cannot see any difference from the moment of inertia taken from the IBM eigenenergies. In the transitional

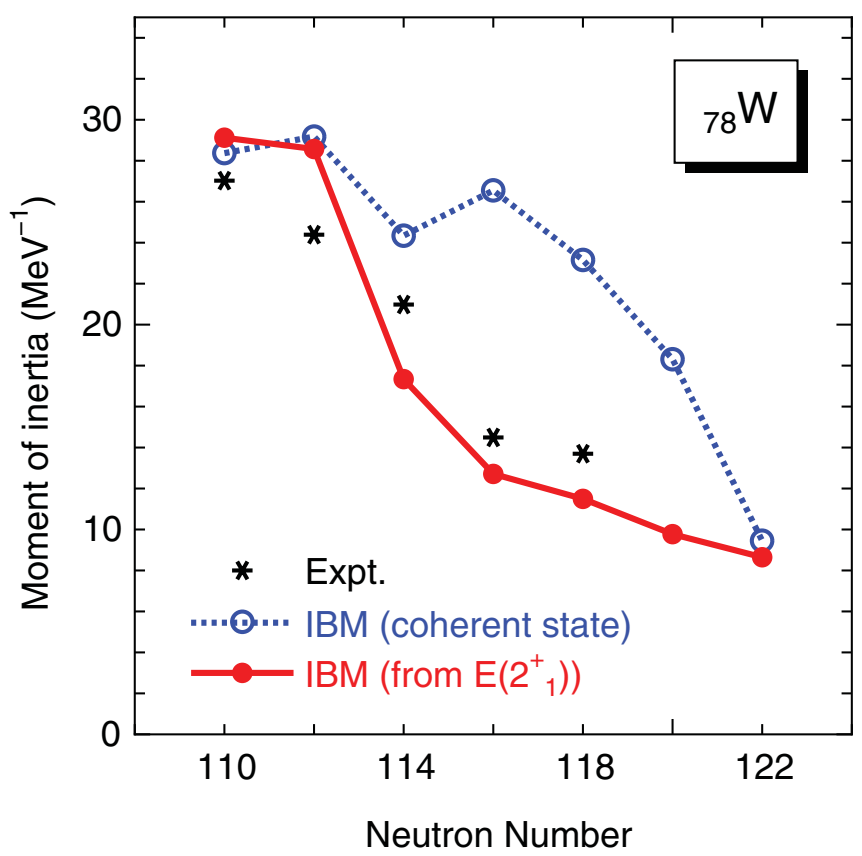

FIG. 3. (Color online) Moments of inertia of W isotopes, computed by the cranking formula for the coherent state, by the the rotor formula $L(L+1)$ using the $2_{1}^{+}$eigenenergies of the IBM and of the experimental $2_{1}^{+}$excitation energies [77]. 
region $114 \leqslant N \leqslant 118$, where according to Fig. 2 a large amount of correlation energy should be involved, however, the moment of inertia of the intrinsic state is far from sufficient and configuration mixing by the diagonalization of the Hamiltonian becomes crucial for the description of the experimental trend.

\section{Excited states}

We now discuss in Figs. 4 and 5 the low-lying states for the considered isotopic chains. Experimentally [77-79], the excitation energies of the ground-state band shown in Fig. 4, namely the $2_{1}^{+}, 4_{1}^{+}, 6_{1}^{+}$, and $8_{1}^{+}$yrast states, increase as the neutron shell closure $N=126$ is approached. The increase of these yrast levels with neutron number $N$ becomes more rapid with smaller $Z$ when departing from the proton shell closure $Z=82$. The present results follow the overall experimental isotopic trend for those nuclei. For $\mathrm{Pt}$, Os, and $\mathrm{W}$ isotopes, the same systematics were observed with the Gogny-D1S functional $[47,53]$.

The LL term has a remarkable influence on the groundstate band at the quantitative level. Without this term, the experimental yrast spectra would not be reproduced with that precision. This is particularly the case with lighter $\mathrm{W}$ (Hf) isotopes with $N=110$ and 112, which follow the rotor formula $L(L+1)$ with their respective experimental ratios being $E_{4_{1}^{+}} / E_{2_{1}^{+}}=3.27$ (3.29) and 3.23 (3.26) [77]. For these nuclei, the results shown in Figs. 4(c) and 4(d) compare rather well with the experiments.

We now turn to the description of the sideband energies in Fig. 5. To begin, we discuss the excited $0^{+}\left(0_{2}^{+}\right)$state. It is well known that the intruder configurations may play a role for midshell $\mathrm{Pt}$ isotopes, where the oblate-prolate shape coexistence is observed [10,63]. The phenomenological IBM study (see Ref. [66], for instance) considers particle-hole excitations across the $Z=82$ proton shell. In this kind of work one needs to extend the boson model space to take into account the intruder configuration with additional proton bosons, arising from (mainly) the $2 p-2 h$ excitation. The normal and the intruder configurations are mixed, and the model Hamiltonian should be then diagonalized in such enlarged configuration space. The validity of this mixing calculation has been discussed extensively $[67,68]$ and is thus of great interest.

The mixing in general becomes more significant when approaching the middle of the major shell. In Fig. 5(a), the
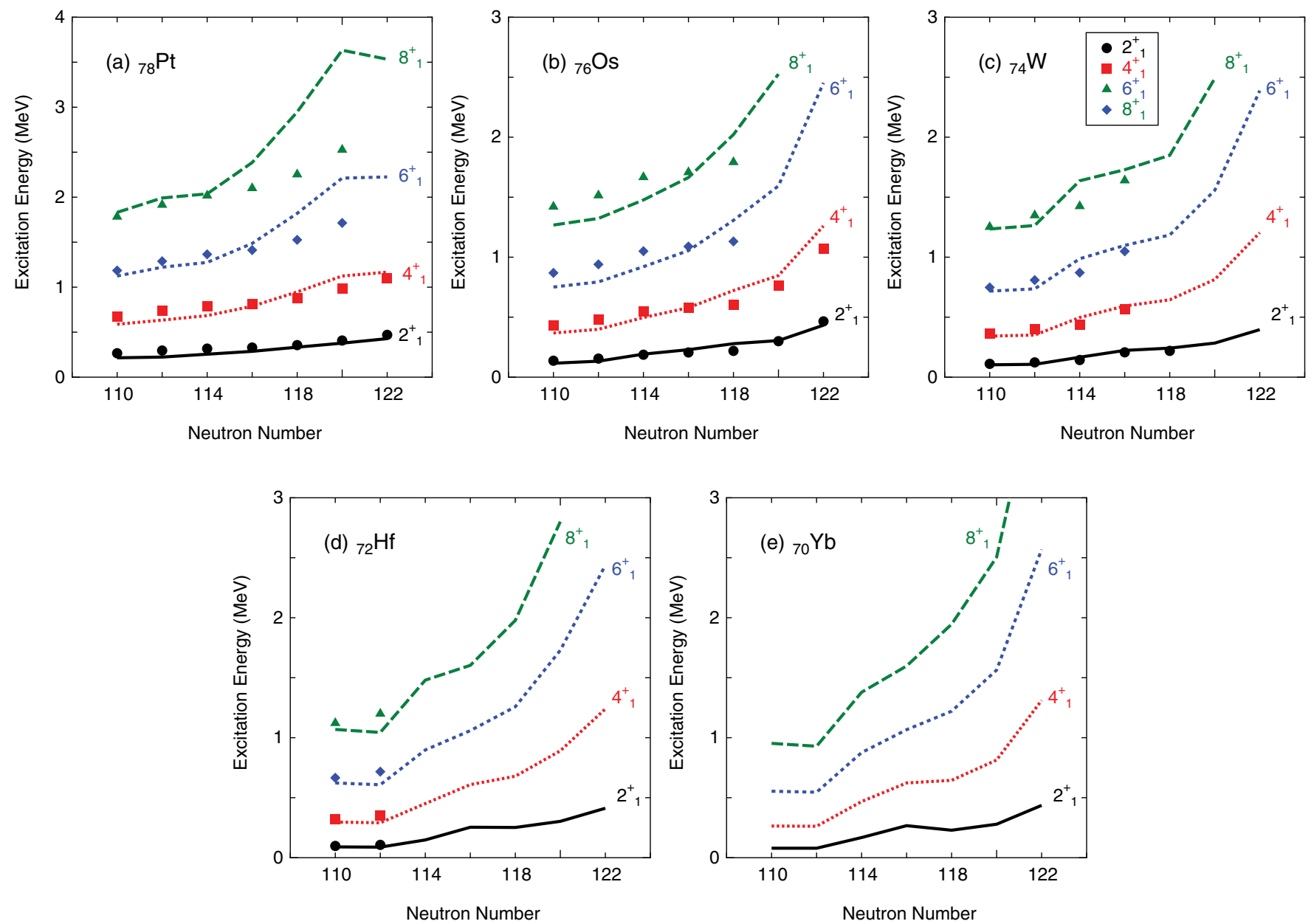

FIG. 4. (Color online) Theoretical (curves) and experimental [77-79] (symbols) low-lying spectra of Yb, Hf, W, Os, and Pt isotopes with $110 \leqslant N \leqslant 122$ for the $2_{1}^{+}, 4_{1}^{+}, 6_{1}^{+}$, and $8_{1}^{+}$states. Symbols for the experimental levels are defined in panel (c). 

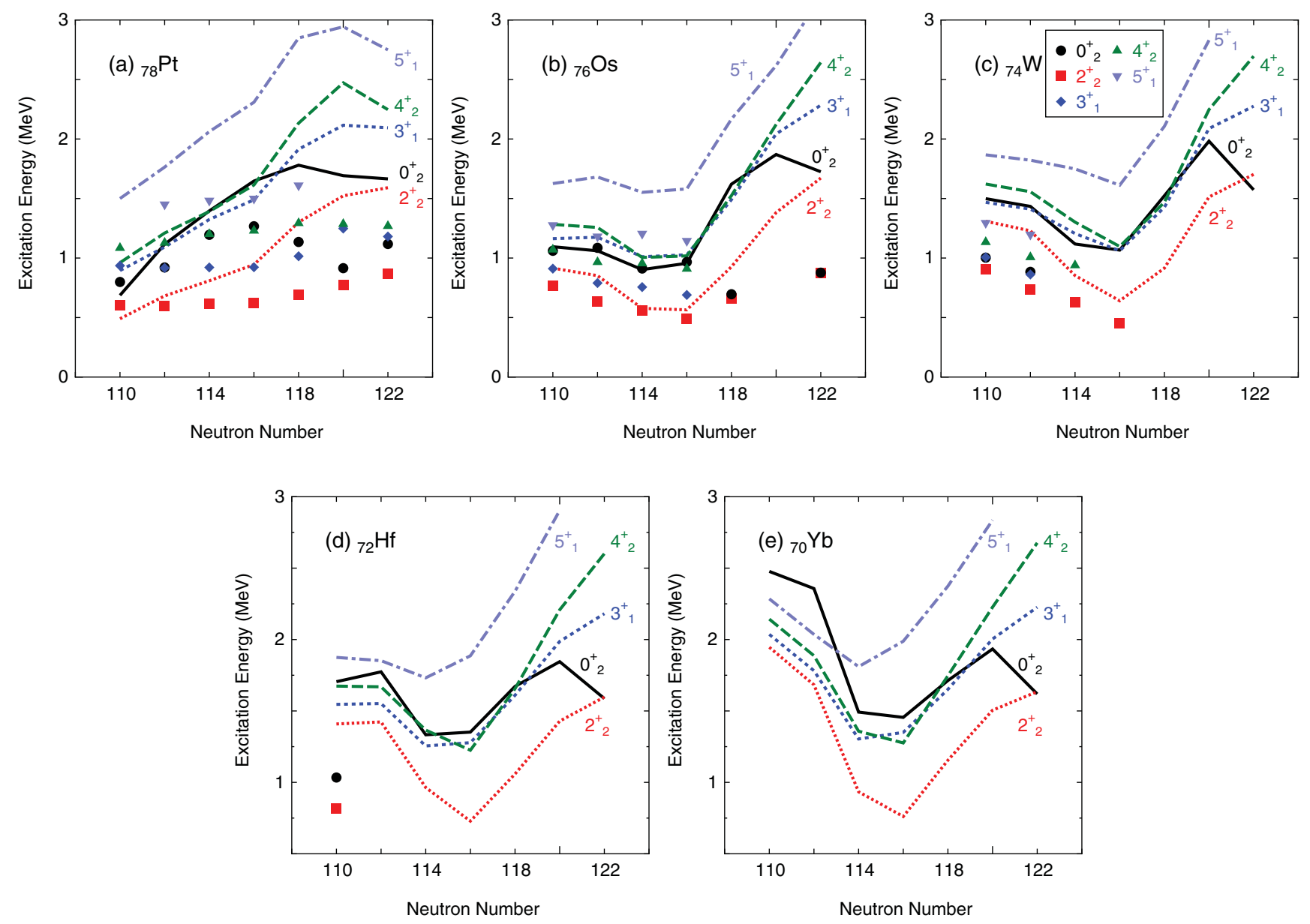

FIG. 5. (Color online) Same as Fig. 4, but for the $0_{2}^{+}, 2_{2}^{+}, 3_{1}^{+}, 4_{2}^{+}$, and $5_{1}^{+}$states.

calculated $0_{2}^{+}$excitation energies for $N \leqslant 116$ Pt isotopes, as well as those with Gogny-D1S [47], seem to compare reasonably well with the data, even without taking into account the mixing between normal and intruder states. Furthermore, the original HFB energy surfaces for Pt isotopes do not exhibit clear coexisting minima. Because of this, the present framework cannot fix the parameters for both the normal and the intruder configurations as well as those for the operators mixing the two configurations. Although such a mixing calculation is a rather subtle problem, it is very interesting to study the extent to which the intruder configuration plays a role when introduced in the present mapping method.

It was shown experimentally [16-19] that, in the nonyrast states of lighter $\mathrm{W}$, Os, and Pt nuclei, the band mixing could arise more or less from the coexistence of the different aforementioned intrinsic states and makes it rather difficult to identify the clear band structure by a model prediction. The band-mixing feature should be outside of the model space of bosons with low spin on which the IBM is built and may be somewhat difficult to be reproduced. It is yet unclear whether the similar complicated band mixing will be observed in the exotic $\mathrm{Yb}$ and $\mathrm{Hf}$ isotopes.

The $2_{2}^{+}$level, which is normally the bandhead of the $K^{\pi}=$ $2^{+}$(so-called quasi- $\gamma$ ) band, is a good test for the evolving triaxiality in a given isotopic chain. Figure 5 shows that the calculated $2_{2}^{+}$level of the $N=116$ nuclei is lowest among each of $\mathrm{Yb}, \mathrm{Hf}, \mathrm{W}$, and Os isotopes. Experimental excitation energies keep steady (decrease) in Pt (Os, W) isotopes as $N$ increases from 110 to 116 .

In our calculations, the decrease of the energies of the $2_{2}^{+}$, $3_{1}^{+}, 4_{2}^{+}$, and $5_{1}^{+}$states occurs more rapidly for lower- $Z$ isotopes, which have a larger number of active bosons. Around $N=116$ a change in this tendency occurs and these excitation energies increase. This is in agreement with the only experimental measurement available in Os isotopes.

A remarkable difference between the theoretical and the experimental quasi- $\gamma$-band structure observed in $\mathrm{Pt}$ and $\mathrm{Os}$ isotopes is that the calculated $3_{1}^{+}$and the $4_{2}^{+}$states, and the $5_{1}^{+}$and $6_{2}^{+}$states as well, form doublets, which are absent in the data. Because all the states in Figs. 5(a) and 5(b) except the $0_{2}^{+}$ones are supposed to be the quasi- $\gamma$-band states, the appearance of these doublets points to the emergence of the $\gamma$-unstable [80] or $\mathrm{O}(6)$ dynamical symmetry [42], in which the spectra belonging to the same family of the quantum number $\tau$ are nearly degenerated. Because the rigid triaxial rotor model with $\gamma=30^{\circ}$ [81] predicts the doublets $\left(2^{+}, 3^{+}\right),\left(4^{+}, 5^{+}\right)$, etc., in the $\gamma$ band, the experimental data for Pt [Fig. 5(a)], Os [Fig. 5(b)], and W isotopes [Fig. 5(c)] 
suggest a situation rather in between the $\gamma$-unstable rotor and the rigid-triaxial rotor pictures. The discrepancy of the $\gamma$-band energies occurs probably because the IBM energy surface does not show the triaxial minimum which is, however, seen in the original HFB energy surface.

There are several possible effects which may eliminate this staggering in the $\gamma$-band spectra and improve the agreement with the experiments at the quantitative level. In the present paper, however, we do not look into the details of this issue due to the large number of additional parameters to be introduced and the lack of experimental data for the $\mathrm{Yb}$ and $\mathrm{Hf}$ nuclei. First, a three-body (cubic) term, which partially breaks $\mathrm{O}(6)$ symmetry, may correct the deviation. This was done mainly in the IBM-1 [74,75]. For the present case some type of cubic term appears to be necessary mainly for W, Os, and Pt nuclei, where the Gogny HFB energy surface exhibits a shallow, but stable, triaxial minimum [56]. Although the calculated excitation energies of the quasi- $\gamma$ band for $\mathrm{Yb}$ and $\mathrm{Hf}$ in Figs. 5(d) and 5(e) look like that of the pure $\mathrm{O}(6)$ limit as well, the validity of this term seems to be marginal in these cases. Indeed for the $\mathrm{Yb}$ and $\mathrm{Hf}$ isotopes the original Gogny-D1M energy surface indicates the discrete change of the minimum point from the oblate $\left(\gamma=60^{\circ}\right)$ to the prolate $\left(\gamma=0^{\circ}\right)$ sides, similarly to the Gogny-D1S energy surface [56].

The second possibility would be to relax the constraint on the deformation parameters $\gamma_{\pi}$ and $\gamma_{\nu}$ so that they could take different values. As the IBM-2 can be viewed as a two-fluid system consisting of proton and neutron bosons, the phase-structure analysis would be exploited in the context of the coherent-state formalism [82], whereas it is not obvious to define a consistent mapping procedure for realistic cases.

The third would be the inclusion of higher-spin bosons, like the $g$ boson. It is not independent of the first possibility involving the cubic term, because the cubic term can be derived effectively from the renormalization of the $g$ boson into the $s d$-boson sector [74]. This would, of course, make the problem more complicated.

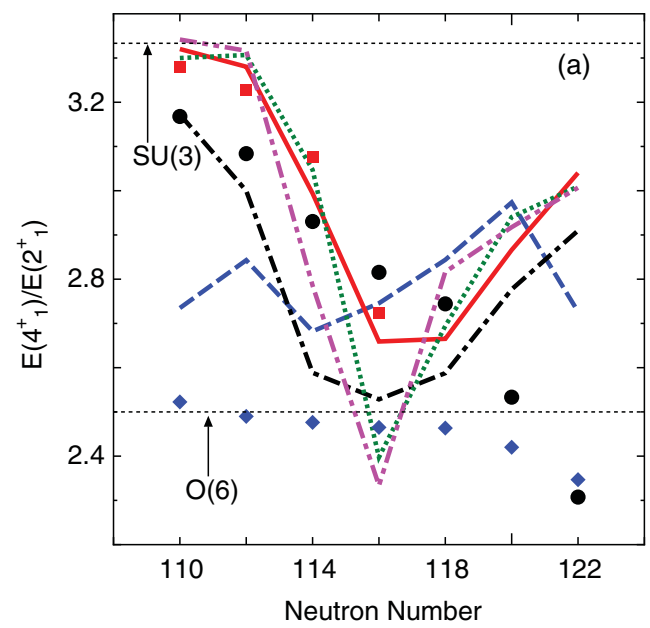

We now address the problem of why the sideband spectra, particularly for Pt and Os isotopes in Figs. 5(a) and 5(b), respectively, are overestimated in the present calculation when approaching the $N=126$ shell closure. The direct reason would be that the microscopic Gogny energy-surface calculation predicts mostly oblate deformations with small quadrupole moment but with a rather large amount of deformation energy characterized by the depth of the potential minimum [56]. Such a topology of the HFB energy surface is not well described by the IBM Hamiltonian close to the end of the major shell $Z=82$. Near the closed shell, one has a relatively small number of bosons. The deviation of the spectra seems to be due to these limited degrees of freedom. The problem in the description of the sideband energies was observed in other cases of shape transitions in different mass regions $[44,46]$ and is still an open problem. According to the preceding argument, it may be expected that the predicted levels for exotic $\mathrm{Yb}$ and $\mathrm{Hf}$ isotopes in the vicinity of the shell closure $N=126$ might be overestimated.

To further examine the problem, it is interesting to consider the relevant energy ratios, because they nicely trace the underlying shape transition. Figure 6 depicts the energy ratios $R_{4 / 2} \equiv E\left(4_{1}^{+}\right) / E\left(2_{1}^{+}\right)\left[\right.$Fig. 6(a)] and $R_{4 \gamma} \equiv E\left(4_{1}^{+}\right) / E\left(2_{2}^{+}\right)$ [Fig. 6(b)] as functions of $N$. The ratio $R_{4 / 2}$ is probably the simplest and best-studied measure for the evolution of collectivity. The ratio $R_{4 \gamma}$ presents the location of the bandhead of the quasi- $\gamma$ band $2_{\gamma}^{+}\left(2_{2}^{+}\right)$relative to the $4_{1}^{+}$excitation energy. Since in many $\gamma$-soft nuclei the $2_{2}^{+}$level lies quite close to the $4_{1}^{+}$level, the overall trend of the ratio $R_{4 \gamma}$ can help to measure the $\gamma$ softness.

In Fig. 6(a), the experimental $R_{4 / 2}$ ratios for Os and W isotopes exhibit a gradual decrease as a function of $N$ from the rotor limit of $R_{4 / 2}=3.3$ in the vicinity of $N=110$ toward the $\mathrm{O}(6)$ limit of $R_{4 / 2}=2.5$. This reflects the transition from the axially deformed rotor to the $\gamma$-unstable shape. Also of particular interest is the difference of the $R_{4 / 2}$ ratio between Pt isotopes and the other isotopes. The experimental $R_{4 / 2}$ ratio

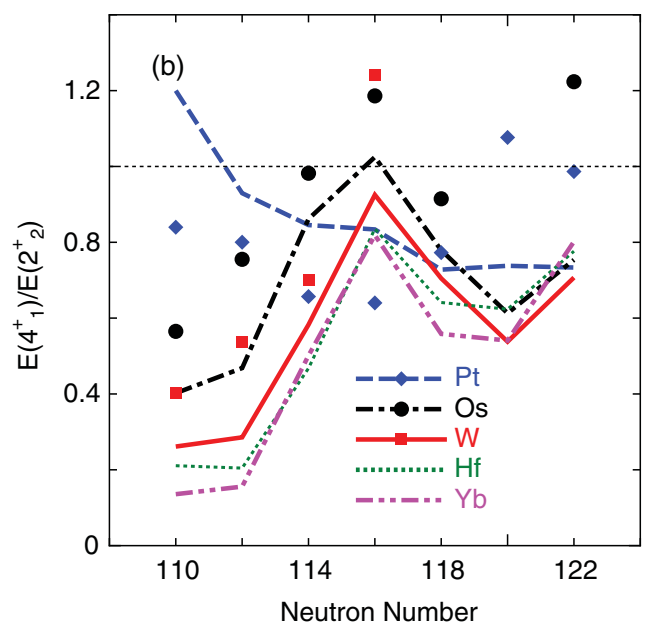

FIG. 6. (Color online) Theoretical (curves) and experimental (symbols) [77] energy ratios (a) $R_{4 / 2}=E\left(4_{1}^{+}\right) / E\left(2_{1}^{+}\right)$and (b) $R_{4 \gamma}=$ $E\left(4_{1}^{+}\right) / E\left(2_{2}^{+}\right)$as functions of $N$. Definitions of the theoretical curves and the symbols for the experimental data appear in panel (b). 
for all the $\mathrm{Pt}$ isotopes studied remains practically constant all the way, being close to the $\mathrm{O}(6)$ limit of 2.5 . The present calculation follows the decrease of the experimental $R_{4 / 2}$ value from $N=110$ to 116 in Os and $\mathrm{W}$ isotopes, whereas an increase is suggested for not only $\mathrm{Os}$ and $\mathrm{W}$ but also $\mathrm{Hf}$ and $\mathrm{Yb}$ isotopes for $N \geqslant 118$, contrary to the experimental tendency of Os isotopes. The change in the calculated ratio $R_{4 / 2}$ occurs quite rapidly for $\mathrm{Hf}$ and $\mathrm{Yb}$ isotopes in comparison to $\mathrm{W}$ and Os isotopes. The discrepancy of the tendency for $N \geqslant 118$ for Os nuclei could be the consequence of the unexpectedly large $\chi_{\pi}$ and $\chi_{\nu}$ values with positive sign, as seen in Table I, since the corresponding IBM energy surfaces exhibit notable oblate deformation. The same would hold for explaining the overall deviation in Pt isotopes. In this context, to describe all the observed data including those for the $N \geqslant 118$ regime, the triaxial dynamics needs to be correctly incorporated in the present model.

The energy ratio $R_{4 \gamma}$ is depicted in Fig. 6(b). The experiment shows that in the lighter $\mathrm{Pt}$, Os, and $\mathrm{W}$ isotopes with $N=110,112$, and 114 , the ratio is less than unity. Whereas for $\mathrm{Pt}$ isotopes the experimental ratio $R_{4 \gamma}$ remains all the way with values close to unity, for Os and $\mathrm{W}$ isotopes the $\gamma$ softness gradually develops with $N$ as the ratio $R_{4 \gamma}$ increases for $110 \leqslant N \leqslant 116$ and bypasses $R_{4 \gamma}=1$ at $N=116$. The overall trend of this experimental ratio for $\mathrm{W}$ and Os isotopes is reproduced in the present calculation, and the same systematic trend is predicted for $\mathrm{Yb}$ and $\mathrm{Hf}$ isotopes. For Os, the experimental $R_{4 \gamma}$ ratio decreases from $N=116$ to 118 , which is reproduced by the calculation. In the heavier isotopes with $N \geqslant 118$ there is a new tendency that the calculated ratio shows an overall decrease, being much less than unity, whereas the experimental ratio for Os isotopes keeps increasing, being larger than unity. The results presented here do not differ much from the case of D1S functional already studied in Refs. [47,53].

\section{E. B(E2) systematics}

Finally, we examine the $B(E 2)$ systematics for a few essential cases corresponding to the shape transition. The $B(E 2)$ ratios relevant to the bandhead of quasi- $\gamma$-band, $2_{2}^{+}$ state can be the stringent tests.

We show in Fig. 7 the ratio $B\left(E 2 ; 2_{2}^{+} \rightarrow 2_{1}^{+}\right) / B\left(E 2 ; 2_{1}^{+} \rightarrow\right.$ $\left.0_{1}^{+}\right)$[Fig. 7(a)] and the branching ratio $B\left(E 2 ; 2_{2}^{+} \rightarrow\right.$ $\left.0_{1}^{+}\right) / B\left(E 2 ; 2_{2}^{+} \rightarrow 2_{1}^{+}\right)$[Fig. 7(b)] for the considered isotopes in comparison with the data $[83,84]$.

The $2_{2}^{+} \rightarrow 2_{1}^{+} E 2$ transition rate shows a certain sensitivity to the neutron number $N$ and thus it is useful as a signature of the structural evolution involving the $\gamma$ softness. The $B\left(E 2 ; 2_{2}^{+} \rightarrow 2_{1}^{+}\right) / B\left(E 2 ; 2_{1}^{+} \rightarrow 0_{1}^{+}\right)$ratios for Pt isotopes differ notably from those of other isotopes. For $\mathrm{Yb}, \mathrm{Hf}, \mathrm{W}$, and Os isotopes, the calculated ratio is peaked at $N=116$. This confirms that in each of these isotopic chains the $N=116$ nucleus is softest in the $\gamma$ direction. On the other hand, for Pt isotopes the calculated $B\left(E 2 ; 2_{2}^{+} \rightarrow 2_{1}^{+}\right) / B\left(E 2 ; 2_{1}^{+} \rightarrow 0_{1}^{+}\right)$ value keeps increasing toward $N=110$ to approach the $\mathrm{O}(6)$ limit, rather than taking a maximum at $N=116$. This tendency appears to be consistent with that expected from the topology of the HFB energy surface [55] and from the predicted systematics of the quasi- $\gamma$ bandhead in Fig. 5(a), which reflects that the $\gamma$ softness persists for rather wide regions in the $\mathrm{Pt}$ isotopic chain.

When compared with the D1S case [53], the present D1M result suggests that the ratio $B\left(E 2 ; 2_{2}^{+} \rightarrow 2_{1}^{+}\right) / B\left(E 2 ; 2_{1}^{+} \rightarrow\right.$ $0_{1}^{+}$) is rather sensitive to the isotopic chains. In fact, in Fig. 7(a), the $B\left(E 2 ; 2_{2}^{+} \rightarrow 2_{1}^{+}\right) / B\left(E 2 ; 2_{1}^{+} \rightarrow 0_{1}^{+}\right)$values below and above $N=116$ appear to have a certain $Z$ dependence when the D1M functional is used. For instance, the $B\left(E 2 ; 2_{2}^{+} \rightarrow\right.$ $\left.2_{1}^{+}\right) / B\left(E 2 ; 2_{1}^{+} \rightarrow 0_{1}^{+}\right)$value for $\mathrm{W}$ isotopes is generally far from the $\mathrm{O}(6)$ limit all the way. It was noticed in Ref. [53], however, that the calculated value of this $B(E 2)$ ratio is practically the same for Os and $\mathrm{W}$ isotopes when the D1S
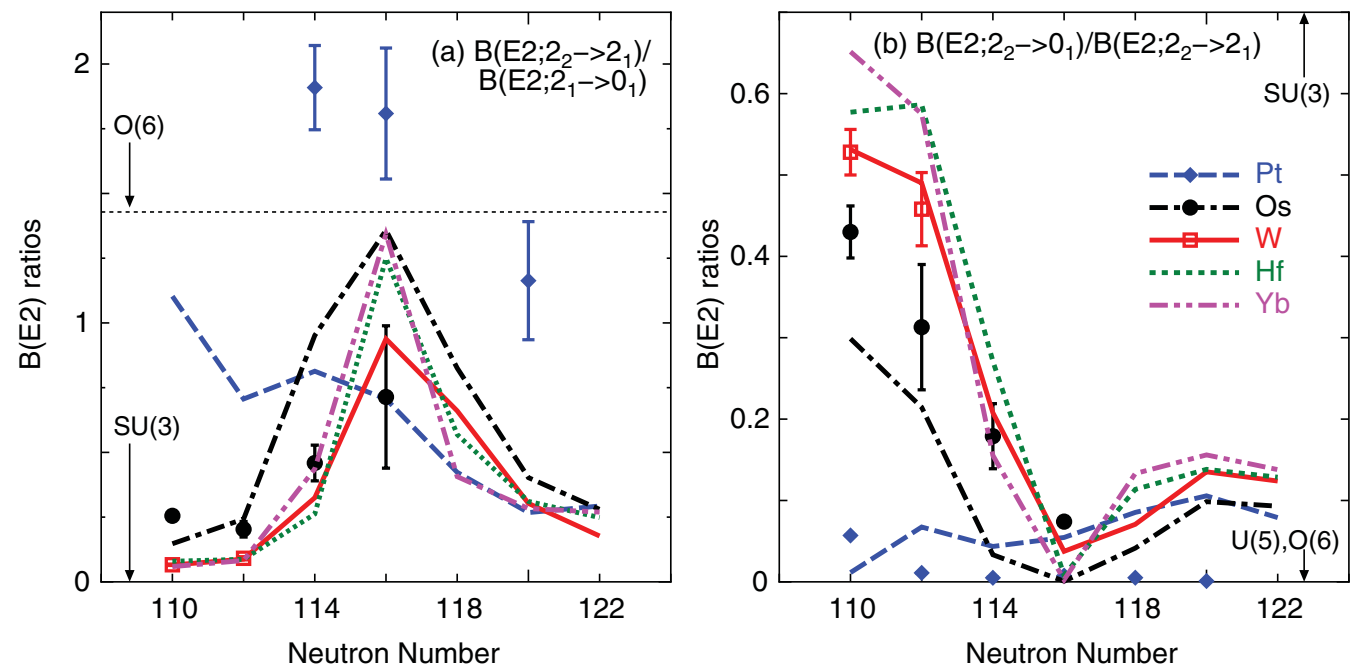

FIG. 7. (Color online) The $B(E 2)$ ratio (a) $B\left(E 2 ; 2_{2}^{+} \rightarrow 2_{1}^{+}\right) / B\left(E 2 ; 2_{1}^{+} \rightarrow 0_{1}^{+}\right)$and the branching ratio (b) $B\left(E 2 ; 2_{2}^{+} \rightarrow 0_{1}^{+}\right) / B\left(E 2 ; 2_{2}^{+} \rightarrow\right.$ $2_{1}^{+}$) for relevant low-lying states of the considered $\mathrm{Yb}, \mathrm{Hf}, \mathrm{W}$, Os, and Pt isotopes with Gogny-D1M EDF. Experimental data for W, Os, and Pt isotopes are taken from Ref. [84]. Definitions of symbols and theoretical curves appear in panel (b). 
functional is taken. It would be interesting to see if this $Z$ dependence is observed experimentally.

The branching ratio $B\left(E 2 ; 2_{2}^{+} \rightarrow 0_{1}^{+}\right) / B\left(E 2 ; 2_{2}^{+} \rightarrow 2_{1}^{+}\right)$in Fig. 7(b) also presents a clear signature of the structural evolution involving triaxiality. For $\mathrm{Yb}, \mathrm{Hf}$, and $\mathrm{W}$ isotopes with $110 \leqslant N \leqslant 116$, the branching ratio decreases from values close to the $\mathrm{SU}(3)$ limit of 0.7 to the $\mathrm{U}(5) / \mathrm{O}(6)$ limit of zero. This behavior corresponds to the transition from well-deformed to $\gamma$-soft nuclei as confirmed by the experimental data on $\mathrm{Os}$ and $\mathrm{W}$ isotopes. At this point, one can observe the increase from $N=116$ toward the shell closure $N=126$. The increase represents the deviation from the $\gamma$-soft character, because the corresponding mapped energy surface in Fig. 1 exhibits notable oblate deformation. The change in the branching ratio occurs more slowly than the D1S case [53]. This is consistent with our general finding that the D1M energy surfaces for these nuclei show less pronounced quadrupole correlation than the D1S ones. As observed in Fig. 7(b), the branching ratios for Pt isotopes remain always much closer to zero, which is compatible with their sustained $\gamma$-soft character.

\section{SUMMARY}

In summary, the method of deriving the Hamiltonian of the interacting boson model from the constrained HFB calculations with the Gogny functional D1M was applied to the spectroscopic analysis of the neutron-rich Yb, Hf, W, Os, and $\mathrm{Pt}$ isotopes. The microscopic energy surface obtained from the constrained HFB calculation turns out to be a good starting point for both reproducing and predicting the ground-state shape of the considered nuclei. Spectroscopic observables that characterize the underlying shape transitions, such as excitation energies, $B(E 2)$ ratios, and correlation energies, were calculated.

It was shown that the Pt isotopes largely differ from the other isotopes in the rapidity of the shape transition. For most of the considered Pt nuclei the mapped IBM energy surfaces are $\gamma$ soft. The transition occurs more rapidly when departing from $Z=76(\mathrm{Os})$ through $Z=70(\mathrm{Yb})$. The triaxial deformation helps to understand the prolate-to-oblate shape transition that occurs in the considered isotopes. The $N=116$ nuclei can be commonly identified as the transition points. This is most noticeably seen in the overall systematic trend of the bandhead of the $\gamma$ band $2_{2}^{+}$, as well as in energy and $B(E 2)$ ratios. Predicted spectra were presented for the neutron-rich $\mathrm{Yb}$ and $\mathrm{Hf}$ isotopes, where a quite rapid structural evolution is suggested. When compared to the results from the standard Gogny-D1S parametrization [47,53], the D1M functional seems to be equally valid to describe the physics involved.

However, the present work aims at investigating the possible ways of refining the current model and clarifying its limitations when applied to the considered mass region. First, as discussed in Sec. IIID, the discrepancy in the level structure of the quasi- $\gamma$ band turns out to be a major limitation. It is likely that this discrepancy is mainly due to the use of the IBM Hamiltonian not reproducing the triaxial energy minimum. A specific three-body (cubic) term may improve the agreement. Second, the boson effective charges need to be determined in a microscopic way and effects beyond the mean field, like core polarization, should be taken into account. It would also be meaningful to compare the spectra and the electromagnetic transition rates resulting from the present method directly with those obtained from full configuration-mixing and symmetry-conserving calculations including triaxial degrees of freedom. This would help to quantify the predictive power of the employed model when applied to heavy exotic nuclei. Work along these directions is in progress.

\section{ACKNOWLEDGMENTS}

This work was supported in part by Grants-in-Aid for Scientific Research (A) No. 20244022 and No. 217368. Author K.N. acknowledges the support by the JSPS. The work of authors R.R., L.M.R, and P.S was supported by MICINN (Spain) under Research Grants No. FIS2008-01301, No. FPA2009-08958, and No. FIS2009-07277, as well as by Consolider-Ingenio 2010 Programs CPAN CSD2007-00042 and MULTIDARK CSD2009-00064. Author R.R. thanks Professor J. Äysto and Professor R. Julin as well as the experimental teams of the University of Jyväskylä (Finland) for warm hospitality and encouraging discussions.
[1] A. Bohr and B. R. Mottelson, Nuclear Structure, Vol. I (Benjamin, New York, 1969); Nuclear Structure, Vol. II (Benjamin, New York, 1975).

[2] P. Ring and P. Schuck, The Nuclear Many-Body Problem (Springer, Berlin, 1980).

[3] J. L. Wood, K. Heyde, W. Nazarewicz, M. Huyse, and P. Van Duppen, Phys. Rep. 215, 101 (1992).

[4] T. R. Werner, J. Dobaczewski, M. W. Guidry, W. Nazarewicz, and J. A. Sheikh, Nucl. Phys. A 578, 1 (1994).

[5] W. Nazarewicz, Nucl. Phys. A 574, 27c (1994).

[6] M. Bender, P.-H. Heenen, and P.-G. Reinhard, Rev. Mod. Phys. 75, 121 (2003).

[7] S. Cwiok, P.-H. Heenen, and W. Nazarewicz, Nature (London) 433, 705 (2005).
[8] R. Rodríguez-Guzmán and P. Sarriguren, Phys. Rev. C 76, 064303 (2007).

[9] P. Sarriguren, R. Rodríguez-Guzmán, and L. M. Robledo, Phys. Rev. C 77, 064322 (2008).

[10] J. L. Egido, L. M. Robledo, and R. R. Rodríguez-Guzmán, Phys. Rev. Lett. 93, 082502 (2004).

[11] L. M. Robledo, R. R. Rodríguez-Guzmán, and P. Sarriguren, Phys. Rev. C 78, 034314 (2008).

[12] I. Hamamoto and B. R. Mottelson, Phys. Rev. C 79, 034317 (2009).

[13] P. Cejnar, J. Jolie, and R. F. Casten, Rev. Mod. Phys. 82, 2155 (2010).

[14] R. Julin, K. Helariutta, and M. Muikku, J. Phys. G 27, R109 (2001). 
[15] G. D. Dracoulis et al., J. Phys. G 12, L97 (1986); Phys. Rev. C 44, R1246 (1991).

[16] P. M. Davidson et al., Nucl. Phys. A 568, 90 (1994).

[17] P. M. Davidson et al., Nucl. Phys A 657, 219 (1999).

[18] T. Kibéti et al., Nucl. Phys. A 567, 183 (1994).

[19] T. Kibéti et al., Nucl. Phys. A 688, 669 (2001).

[20] C. Y. Wu et al., Nucl. Phys A 607, 178 (1996).

[21] Zs. Podolyák et al., Phys. Lett. B 491, 225 (2000).

[22] M. Pfützner et al., Phys. Rev. C 65, 064604 (2002).

[23] M. Caamaño et al., Eur. Phys. J. A 23, 201 (2005).

[24] R. Rodríguez-Guzmán, P. Sarriguren, L. M. Robledo, and S. Perez-Martín, Phys. Lett. B 691, 202 (2010).

[25] R. Rodríguez-Guzmán, P. Sarriguren, and L. M. Robledo, Phys. Rev. C 82, 044318 (2010).

[26] R. Rodríguez-Guzmán, P. Sarriguren, and L. M. Robledo, Phys. Rev. C 82, 061302(R) (2010).

[27] N. Schunck, J. Dobaczewski, J. McDonnell, J. More, W. Nazarewicz, J. Sarich, and M. V. Stoitsov, Phys. Rev. C 81, 024316 (2010).

[28] K. J. Pototzky et al., Eur. Phys. J. A 46, 299 (2010).

[29] T. H. R. Skyrme, Nucl. Phys. 9, 615 (1959).

[30] D. Vautherin and D. M. Brink, Phys. Rev. C 5, 626 (1972).

[31] J. Decharge, M. Girod, and D. Gogny, Phys. Lett. B 55, 361 (1975),

[32] J. Dechargé and D. Gogny, Phys. Rev. C 21, 1568 (1980).

[33] T. Nikšić, D. Vretenar, and P. Ring, Prog. Part. Nucl. Phys. 66, 519 (2011)

[34] M. Bender, G. F. Bertsch, and P.-H. Heenen, Phys. Rev. C 73, 034322 (2006).

[35] M. Bender and P.-H. Heenen, Phys. Rev. C 78, 024309 (2008).

[36] R. R. Rodríguez-Guzmán, J. L. Egido, and L. M. Robledo, Phys. Rev. C 69, 054319 (2004).

[37] R. Rodríguez-Guzmán, J. L. Egido, and L. M. Robledo, Nucl. Phys. A 709, 201 (2002).

[38] J. M. Yao, H. Mei, H. Chen, J. Meng, P. Ring, and D. Vretenar, Phys. Rev. C 83, 014308 (2011).

[39] P. Bonche, J. Dobaczewski, H. Flocard, P.-H. Heenen, and J. Meyer, Nucl. Phys. A 510, 466 (1990).

[40] Z. P. Li, T. Nikšić, D. Vretenar, and J. Meng, Phys. Rev. C 81, 034316 (2010); Z. P. Li, T. Nikšić, D. Vretenar, J. Meng, G. A. Lalazissis, and P. Ring, ibid. 79, 054301 (2009).

[41] J.-P. Delaroche, M. Girod, J. Libert, H. Goutte, S. Hilaire, S. Peru, N. Pillet, and G. F. Bertsch, Phys. Rev. C 81, 014303 (2010).

[42] F. Iachello and A. Arima, The Interacting Boson Model (Cambridge University Press, Cambridge, UK, 1987).

[43] T. Otsuka, A. Arima, and F. Iachello, Nucl. Phys. A 309, 1 (1978).

[44] K. Nomura, N. Shimizu, and T. Otsuka, Phys. Rev. Lett. 101, 142501 (2008).

[45] T. Mizusaki and T. Otsuka, Prog. Theor. Phys., Suppl. 125, 97 (1997).

[46] K. Nomura, N. Shimizu, and T. Otsuka, Phys. Rev. C 81, 044307 (2010).

[47] K. Nomura, T. Otsuka, R. Rodríguez-Guzmán, L. M. Robledo, and P. Sarriguren, Phys. Rev. C 83, 014309 (2011).

[48] T. Otsuka, Phys. Rev. Lett. 46, 710 (1981); Nucl. Phys. A 368, 244 (1981).

[49] T. Otsuka, Phys. Lett. B 138, 1 (1984).

[50] T. Otsuka and J. N. Ginocchio, Phys. Rev. Lett. 55, 276 (1985).

[51] T. Otsuka and N. Yoshinaga, Phys. Lett. B 168, 1 (1986).
[52] K. Nomura, T. Otsuka, N. Shimizu, and L. Guo, Phys. Rev. C 83, 041302(R) (2011).

[53] K. Nomura, T. Otsuka, R. Rodríguez-Guzmán, L. M. Robledo, P. Sarriguren, P. H. Regan, P. D. Stevenson, and Zs. Podolyák, Phys. Rev. C 83, 054303 (2011).

[54] J. F. Berger, M. Girod, and D. Gogny, Nucl. Phys. A 428, 23c (1984).

[55] R. Rodríguez-Guzmán, P. Sarriguren, L. M. Robledo, and J. E. Garcia-Ramos, Phys. Rev. C 81, 024310 (2010).

[56] L. M. Robledo, R. Rodríguez-Guzmán, and P. Sarriguren, J. Phys. G: Nucl. Part. Phys. 36, 115104 (2009).

[57] S. Goriely, S. Hilaire, M. Girod, and S. Peru, Phys. Rev. Lett. 102, 242501 (2009).

[58] J. Jolie and A. Linnemann, Phys. Rev. C 68, 031301(R) (2003).

[59] J. A. Cizewski, R. F. Casten, G. J. Smith, M. L. Stelts, W. R. Kane, H. G. Borner, and W. F. Davidson, Phys. Rev. Lett. 40, 167 (1978).

[60] R. F. Casten and J. A. Cizewski, Nucl. Phys. A 309, 477 (1978).

[61] Zs. Podolyák et al., Phys. Rev. C 79, 031305(R) (2009).

[62] T. Nikšić, P. Ring, D. Vretenar, Tuan Tian, and Zhong-yu Ma, Phys. Rev. C 81, 054318 (2010).

[63] A. N. Andreyev et al., Nature (London) 405, 430 (2000).

[64] T. Duguet, M. Bender, P. Bonche, and P.-H. Heenen, Phys. Lett. B 559, 201 (2003).

[65] M. Bender, P. Bonche, T. Duguet, and P.-H. Heenen, Phys. Rev. C 69, 064303 (2004).

[66] P. D. Duval and B. R. Barrett, Nucl. Phys. A 376, 213 (1983).

[67] E. A. McCutchan, R. F. Casten, and N. V. Zamfir, Phys. Rev. C 71, 061301(R) (2005).

[68] J. E. Garcia-Ramos and K. Heyde, Nucl. Phys. A 825, 39 (2009).

[69] A. E. L. Dieperink, O. Scholten, and F. Iachello, Phys. Rev. Lett. 44, 1747 (1980).

[70] D. J. Thouless and J. G. Valatin, Nucl. Phys. 31, 211 (1962).

[71] D. R. Inglis, Phys. Rev. 103, 1786 (1956).

[72] S. T. Belyaev, Nucl. Phys. 24, 322 (1961).

[73] H. Schaaser and D. M. Brink, Phys. Lett. B 134, 269 (1984).

[74] K. Heyde, P. Van Isacker, M. Waroquier, and J. Moreau, Phys. Rev. C 29, 1420 (1984).

[75] R. F. Casten, P. von Brentano, K. Heyde, P. Van Isacker, and J. Jolie, Nucl. Phys. A 439, 289 (1985).

[76] R. Rodríguez-Guzmán, Y. Alhassid, and G. F. Bertsch, Phys. Rev. C 77, 064308 (2008).

[77] NuDat 2.5 [http://www.nndc.bnl.gov/nudat2/index.jsp].

[78] N. Alkhomashi et al., Phys. Rev. C 80, 064308 (2009).

[79] P. H. Regan et al., Int. J. Mod. Phys. E 17, 8 (2008).

[80] L. Wilets and M. Jean, Phys. Rev. 102, 788 (1956).

[81] A. S. Davydov and G. F. Filippov, Nucl. Phys. 8, 237 (1958).

[82] M. A. Caprio and F. Iachello, Ann. Phys. (NY) 318, 454 (2005).

[83] The experimental $B(E 2)$ value for the $2_{2}^{+} \rightarrow 2_{1}^{+}$transition in the ${ }^{194} \mathrm{Pt}$ nucleus has been updated since the one presented in Fig. 4(a) of Ref. [47]. Thus, the present work presents a more complete compilation of the experimental $B(E 2)$ data, taken from Ref. [84].

[84] S.-C. Wu and H. Niu, Nucl. Data Sheets 100, 483 (2003); B. Singh and R. B. Firestone, ibid. 74, 383 (1995); C. M. Baglin, ibid. 111, 275 (2010); 99, 1 (2003); B. Singh, ibid. 95, 387 (2002); 99, 275 (2003); C. M. Baglin, ibid. 84, 717 (1998); B. Singh, ibid. 107, 1531 (2006); H. Xiaolong, ibid. 108, 1093 (2007); 110, 2533 (2007); F. G. Kondeva and S. Lalkovski, ibid. 108, 1471 (2007). 